\title{
Age-dependent sensitivity of the mouse kidney to chronic nicotine exposure
}

\author{
Istvan Arany ${ }^{1}$, Samuel Hall ${ }^{1}$ and Mehul Dixit ${ }^{1}$
}

BACKGROUND: Many adolescents are exposed to nicotine via smoking, e-cigarette use, or second-hand smoke. Nicotineinduced renal oxidative stress and its long-term consequences may be higher in adolescents than in adults because of intrinsic factors in the adolescent kidney.

METHODS: Adolescent and adult male C57B//6J mice were subjected to 2 or $200 \mu \mathrm{g} / \mathrm{ml}$ nicotine, which closely emulates passive or active smoking, respectively, for 4 weeks. Extent of nicotine exposure (cotinine content), oxidative stress (HNE), renal function (creatinine), tubular injury (KIM-1), and pretreatment renal levels of select pro-oxidant (p66shc) and antioxidant (Nrf2/MnSOD) genes were determined. Impact of p66shc overexpression or Nrf2/MnSOD knockdown on low-/high-dose nicotine-induced oxidative stress was determined in cultured renal proximal tubule cells.

RESULTS: Despite similar plasma/renal cotinine levels, renal HNE and KIM-1 contents were higher in adolescents compared with those in adults, whereas renal function was unaltered after passive or active smoking-equivalent nicotine exposure. Pretreatment levels of p66shc were higher, whereas $\mathrm{Nrf2/MnSOD}$ levels were lower in the adolescent kidney. In agreement with this, overexpression of p66shc or knockdown of Nrf2/MnSOD augmented nicotine-induced ROS production in renal proximal tubule cells.

CONCLUSION: Chronic nicotine exposure incites higher oxidative stress in the adolescent than in adult kidney because of a pre-existent pro-oxidant milieu.

A dverse effects of smoking on renal health have been recognized for more than a decade (1). It has been shown that nicotine (NIC) - a major tobacco alkaloid (2)-links smoking to renal injury (3) via increased oxidative stress (4). Although the overall rate of cigarette smoking has declined in the United States among adolescents (5), there is a major increase in use of alternative NIC delivery products such as hookah, smokeless tobacco, and e-cigarettes (5). Perceived as safe alternative to cigarettes (6) the popularity of e-cigarettes is alarmingly increasing, especially among adolescents (5), which may lead to a "pediatric epidemic" (7) with potentially increasing renal risk in the adolescent population (8). In addition to e-cigarette use, adolescents are also exposed to second-hand smoke by smoking parents (9). Studies revealed that urinary and plasma cotinine-a stable NIC metabolite (2) - content in nonsmoking children exposed to parental tobacco smoke is higher than in those who were not exposed to parental tobacco smoke (10), and it correlates with the number of cigarettes smoked per day by the parent (11). Thus, children/adolescents are probably also at high risk to secondhand smoke-related health problems that could also entail increased renal risk (9).

Studies indicate that expression of oxidant-handling genes is developmentally regulated; select antioxidant genes are lower in the developing rat kidney (12), whereas levels of $\mathrm{H}_{2} \mathrm{O}_{2}$-producing enzymes are higher in the chick kidney (13) compared with those in the adult kidney. As NIC exerts its adverse effects via induction of oxidative stress (3), developmental status of oxidant-handling enzymes may influence sensitivity of the kidney to chronic NIC exposure.

Accordingly, the aim of this study was to determine whether the adolescent kidney is more vulnerable to oxidative stress imposed by passive or active smoking-equivalent NIC exposure than the adult kidney in mice. We also sought to establish a correlation between an imbalanced pro-oxidant and antioxidant system in the adolescent kidney and the response to nicotine exposure. In addition, in vitro studies were designed to recapitulate the in vivo findings in cultured renal proximal tubule cells.

\section{METHODS}

\section{Animal Studies}

Wild-type male C57Bl/6J mice (Jackson Laboratories, Bar Harbor, $\mathrm{ME}$ ) at various ages (4 and 24 weeks) were used. 4-week-old age corresponds to early adolescence, whereas 24-week-old age corresponds to early adulthood in mice (14). Some animals at both age groups (6-6) were exposed to $200 \mu \mathrm{g} / \mathrm{ml} \mathrm{NIC} \mathrm{in} 2 \%$ saccharine or vehicle for 4 weeks ("active smoking"-equivalent). This amount of NIC exposure results in similar levels of cotinine in the plasma of mice as that found in longtime active smokers as we described elsewhere (4). Other groups were exposed to $2 \mu \mathrm{g} / \mathrm{ml} \mathrm{NIC} \mathrm{in} 2 \%$ saccharine ("passive smoking"-equivalent) or vehicle for 4 weeks. This amount of NIC exposure results in closely similar levels of cotinine (up to $2 \mathrm{ng} / \mathrm{ml}$ ) in the plasma of mice as that found in pediatric age groups exposed to second-hand smoke (15) (Supplementary Figure S1). All procedures were approved by the 


\section{Nicotine and the adolescent kidney Articles}

Institutional Animal Care and Use Committee of the University of Mississippi Medical Center.

\section{Plasma Cotinine Assay}

Plasma cotinine content was determined by a Cotinine ELISA kit (Calbiotech, Spring Valley, CA) according to the manufacturer's recommendation.

\section{Determination of Renal Oxidative Stress}

Extent of renal oxidative stress was determined from kidney lysates using the OxiSelect HNE-His adduct ELISA kit (Cell Biolabs,, San Diego, CA). Values were normalized to protein content of the lysates.

\section{Determination of Renal Dysfunction and Tubular Injury}

Renal dysfunction was determined using the Quantichrom Creatinine Assay kit (BioAssay Systems, Hayward, CA) as recommended by the manufacturer.

Renal KIM-1 content-a marker of renal tubular injury (16) was determined from kidney lysates using the "Quantikine mouse TIM-1/KIM-1/HAVCR immunoassay” kit (R\&D Systems, Minneapolis, MN).

\section{Preparation of Lysates and Western Blotting}

Preparation of kidney lysates and Western blotting were similar to that we described elsewhere (4). The anti-Nrf2 and anti-MnSOD (SOD2) antibodies were purchased from Santa Cruz Biotechnology (Santa Cruz, CA), the anti-p66shc antibody from Nanotools/Axxora (San Diego, CA), and the secondary antibodies were purchased from Cell Signaling Technologies (Danvers, MA). Equal loading was determined by rehybridization of the blots with actin (Millipore, Billerica, MA) after stripping. Bands were visualized by Pierce ECL Western blotting substrate (Thermo Scientific, Rockford, IL) and exposed to an X-ray film (Midwest Scientific, St. Louis, MO). Films were digitized and analyzed by the Un-Scan-It Version 6.1 software (Silk Scientific, Orem, UT).

\section{Cell Line and Treatments}

The renal proximal tubule cell line (NRK52E) was purchased from ATCC (Manassas, VA) and maintained in DMEM medium supplemented with $10 \%$ fetal bovine serum in $5 \% \mathrm{CO}_{2}$ atmosphere at $37^{\circ} \mathrm{C}$. Semiconfluent cells were treated with 2 or $200 \mu \mathrm{m} / \mathrm{l}$ nicotine as described in the relevant section.

\section{Transfection of Cells}

Semiconfluent NRK52E cells were transfected with a p66shc overexpressing plasmid as described earlier (17). Nrf2 expression was knocked down via transfecting cells with $50 \mathrm{~nm}$ Nrf2 siRNA (Santa Cruz) using Lipofectamine 3000 (Life Technologies, Grand Island, NY). To knockdown MnSOD expression, NRK52E cells were transfected with a short-hairpin MnSOD plasmid (Addgene, Cambridge, MA) using Lipofectamine 3000, as described elsewhere. The extent of p66shc overexpression and MnSOD knockdown was confirmed by Western blotting (18). Nrf2 knockdown is verified by determining the activity of the Nrf2-binding antioxidant response element in a reporter luciferase assay (Supplementary Fig S2).

\section{Determination of Intracellular ROS Production In Vitro}

Cells grown in T25 flasks were transfected-if needed-as described above. After $24 \mathrm{~h}$, cells were trypsinized and loaded with $100 \mu \mathrm{m}$ of the oxidant-sensitive $2^{\prime}, 7^{\prime}$-dichlorofluorescein-diacetate (DCFDALife Technologies) for $30 \mathrm{~min}$ at $37^{\circ} \mathrm{C}$. After washing, equal amounts $\left(0.2 \times 10^{6}\right)$ of cells were aliquoted in a 96-well plate and ROS production was determined in the presence or absence of 2 or $200 \mu \mathrm{M}$ NIC (4). ROS production was calculated as changes in fluorescence/ $30 \mathrm{~min} / 0.2 \times 10^{6}$ cells and expressed as a percentage of the corresponding untreated values, as described elsewhere (17).
Statistical Analysis

Continuous variables are expressed as means and standard deviations (SD). One-way ANOVA with Holm-Sidak post-hoc test was used to evaluate the differences between groups. Differences between means were considered significant if $P<0.05$. All analyses were performed using the GraphPad InStat 3.0 (GraphPad Software, La Jolla, CA) software package.

\section{RESULTS}

Chronic Nicotine Exposure Results in Higher Renal Oxidative Stress and Injury but not Renal Dysfunction in Adolescent than in Adult Mice

4- or 24-week-old mice were exposed to either 2 or $200 \mu \mathrm{g} / \mathrm{ml}$ NIC in their drinking water for 4 weeks. Control mice received vehicle. Renal oxidative stress was determined by measuring HNE content in kidney lysates and normalized to the protein content of the lysates. Figure 1a demonstrates that $2 \mu \mathrm{g} / \mathrm{ml}$ NIC exposure (which closely emulates passive smoking-equivalent NIC exposure) resulted in a moderate but significant increase in renal HNE content in the adolescent but not in the adult kidney. In contrast, $200 \mu \mathrm{g} /$ ml NIC exposure (which emulates active smoking) significantly elevated renal HNE content both in adolescents and adults (Figure 1b). Interestingly, the increase was significantly higher in adolescents than in adults. It is important to note that renal HNE content was also significantly higher in the vehicle-exposed adolescent kidneys than in the vehicleexposed adult kidneys (Figure 1a,b). The observed higher oxidative stress was also accompanied by higher expression of KIM-1 (a marker of renal tubular injury (16)) in the adolescent kidney (Figure 1c,d). Importantly, these changes did not lead to any renal dysfunction, as determined by measuring plasma creatinine levels (Figure 1e,f). These results suggest that (1) the untreated adolescent kidney shows a higher level of oxidative stress than the adult kidney; (2) the adolescent, but not the adult kidney is sensitive to passive smoking-equivalent NIC exposure; (3) the adolescent kidney is more sensitive to active smoking-equivalent NIC exposure than the adult kidney, and (4) NIC-induced oxidative stress leads to some degree of tubular injury without resulting in renal dysfunction.

Plasma and Renal Cotinine Contents are Similar in Adolescent and Adult Mice after Chronic Nicotine Exposure

Results presented in Figure 1 raised the possibility that chronic NIC exposure may result in higher NIC level in adolescents than in adults, which may account for the higher oxidative stress. Accordingly, cotinine levels-a marker of NIC exposure (2)-were determined in the plasma and kidneys of mice. According to our results, cotinine content of the adolescent plasma is not different from cotinine content of the adult plasma after passive (Figure 2a) or active (Figure 2b) smoking-equivalent NIC exposure. Similarly, the adolescent kidney does not accumulate more cotinine than the adult kidney does (Figure 2c,d). These results imply that the higher sensitivity of the adolescent kidney to chronic 


\section{Articles | Arany et al.}

NIC exposure is not associated with higher NIC accumulation in the plasma or kidney.

\section{Outcome of Nicotine Exposure Correlates with Pretreatment Levels of Select Antioxidant and Pro-oxidant Genes}

Some animal studies suggest the presence of an immature oxidant-handling system in the adolescent kidney $(12,13)$, which may account for the observed higher oxidative stress in response to chronic NIC exposure (Figure 1). Accordingly, we determined pretreatment levels of select antioxidant (Nrf2 and MnSOD) and pro-oxidant (p66shc) genes in the adolescent kidney by Western blotting and compared them with the levels in the adult kidney. Figure 3 shows that the expression of these genes is developmentally regulated in

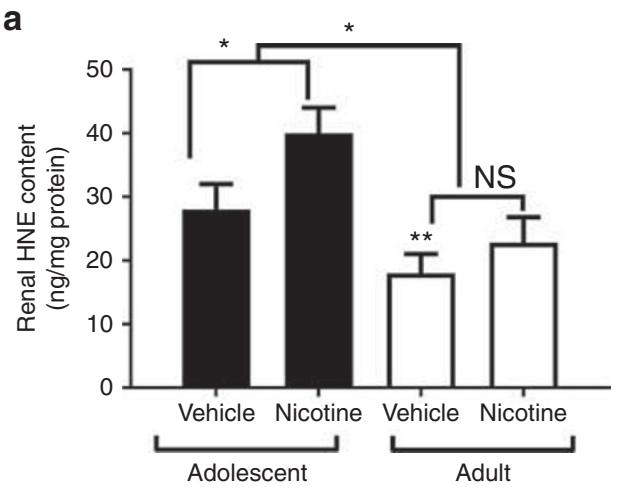

b

C

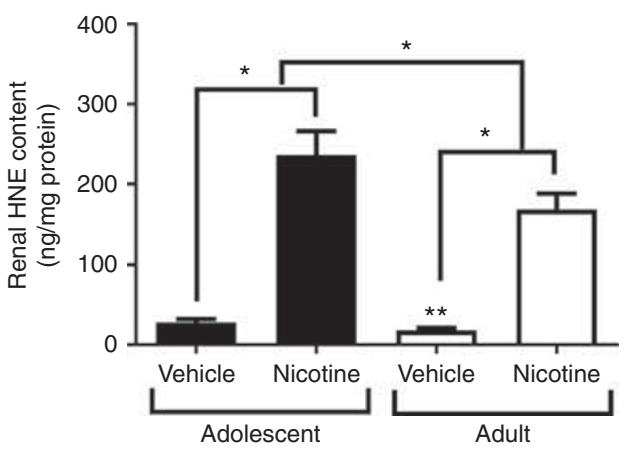

d
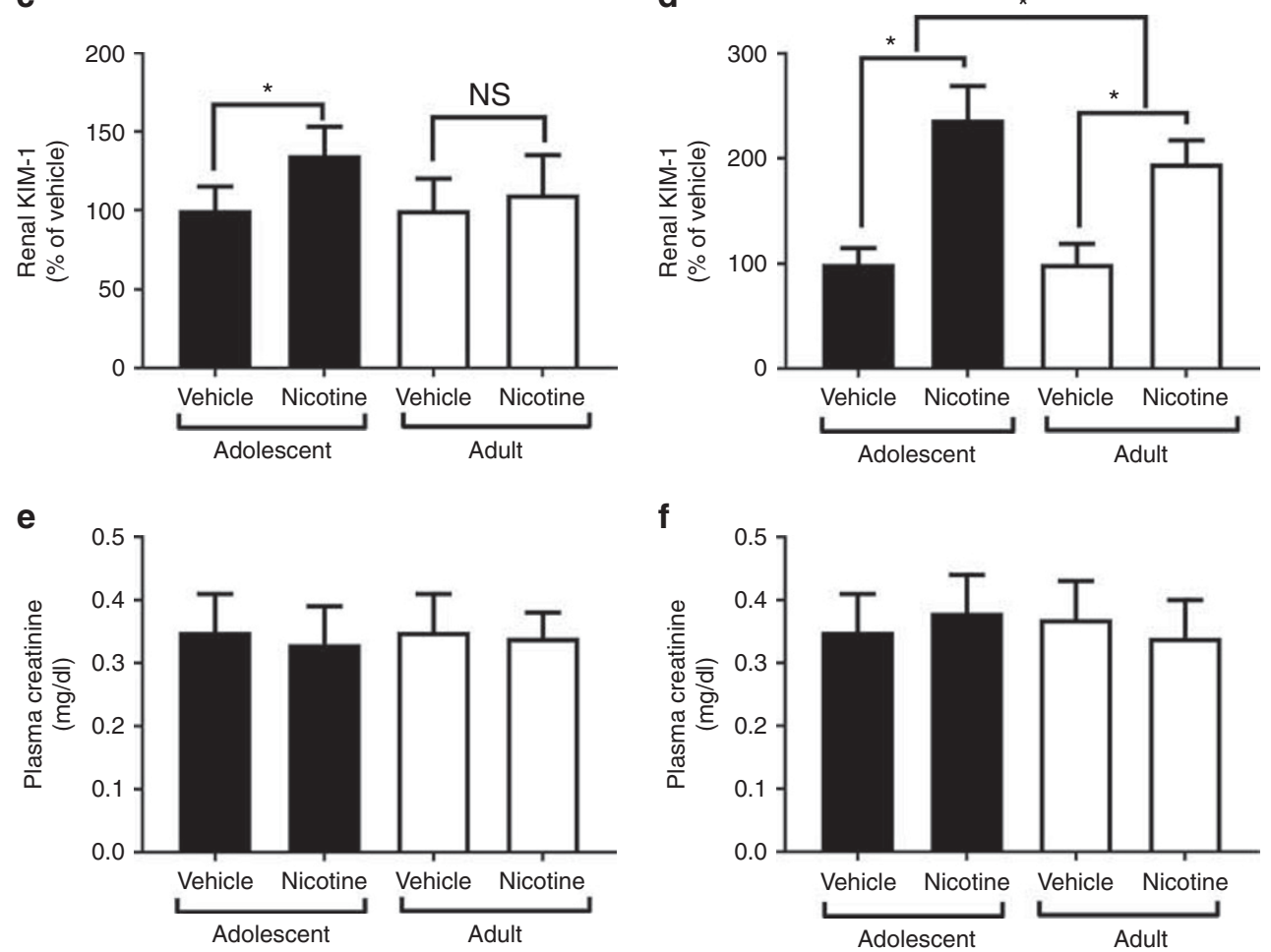

Figure 1. Renal oxidative stress and tubular injury is higher in the adolescent than in the adult kidney after passive or active smoking-equivalent nicotine exposure in mice. (a) 4-week-old or 24-week-old male C57Bl/6J mice were exposed to $2 \mu \mathrm{g} / \mathrm{ml}$ nicotine (passive smoking) or vehicle (2\% saccharine) via their drinking water for 4 weeks. Renal oxidative stress was determined by measuring HNE content (a lipid peroxidation product), as described in the Methods section. $n=6,{ }^{*} P<0.05$ as indicated; ${ }^{* *} P<0.05$ compared with vehicle in adolescents. (b) 4-week-old or 24-week-old male C57Bl/6J mice were exposed to $200 \mu \mathrm{g} / \mathrm{ml}$ nicotine (active smoking) or vehicle (2\% saccharine) via their drinking water for 4 weeks. Renal oxidative stress was determined by measuring a lipid peroxidation product (HNE content), as described in the Methods section. $n=6,{ }^{*} P<0.05$ as indicated; ${ }^{* *} P<0.05$ compared with vehicle in adolescents. (c) Extent of tubular injury was determined by measuring KIM- 1 content of kidney lysates from adolescent and adult mice treated with passive smoking-equivalent NIC. These lysates are identical to those in (a); $n=6,{ }^{*} P<0.05$ as indicated. (d) Extent of tubular injury was determined by measuring KIM-1 content of kidney lysates from adolescent and adult mice treated with active smokingequivalent NIC. These lysates are identical to those in (b); $n=6,{ }^{*} P<0.05$ as indicated. (e) Extent of renal dysfunction was determined by measuring plasma creatinine in adolescent and adult mice treated with passive smoking-equivalent NIC. Plasma derived from the same mice as in (a); $n=6$. (f) Extent of renal dysfunction was determined by measuring plasma creatinine in adolescent and adult mice treated with active smoking-equivalent NIC. Plasma derived from the same mice as in $(\mathbf{b}) ; n=6$. NS, not significant. 
a

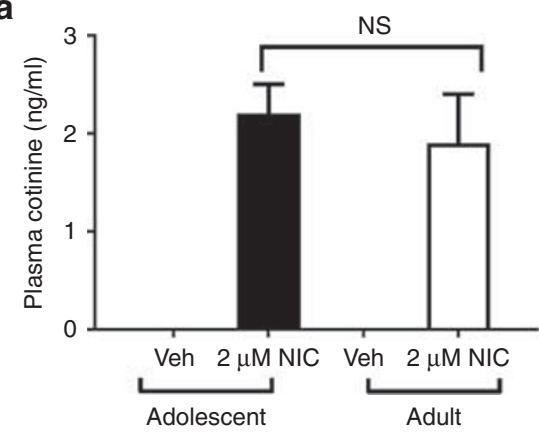

b

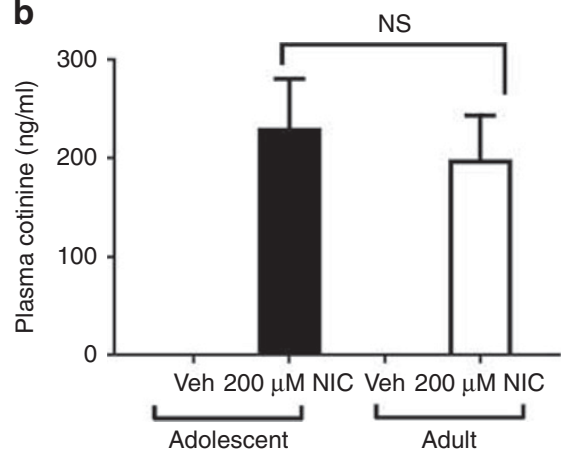

C

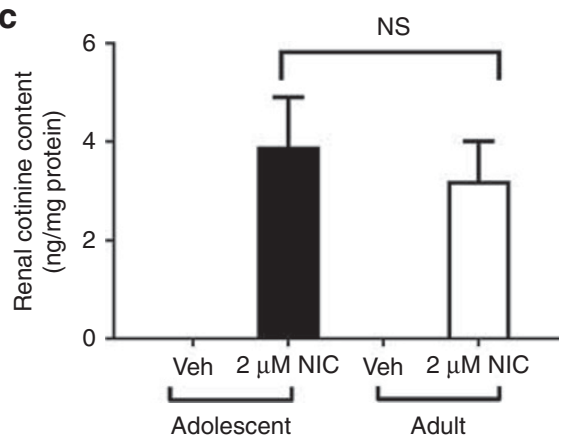

d

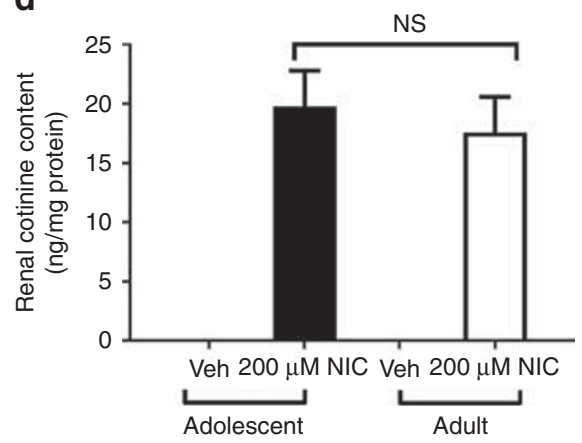

Figure 2. Cotinine content of the plasma and kidney is similar in adolescent and adult mice. Cotinine content of the plasma in adolescent and adult mice treated with passive (a) or active (b) smoking-equivalent nicotine was determined. Cotinine content of the kidney was also determined after treatment with passive (c) or active (d) smoking-equivalent nicotine and normalized to protein content of the lysate. $n=6$. These mice are the same as in Figure 1. NS, not significant; veh, vehicle.

mice; whereas the levels of Nrf2 and MnSOD are lower in the adolescent kidney, and the expression of p66shc is higher than that in the adult kidney. These results suggest a correlation between NIC-induced oxidative stress and high levels of select pro-oxidant and low levels of select antioxidant genes in the mouse kidney.

\section{High Levels of p66shc and Deficiency in Nrf2 or MnSOD} Augment Nicotine-Induced Oxidative Stress in Cultured Renal Proximal Tubule Cells

Our in vivo results suggest that a more pro-oxidant milieu in the adolescent kidney may be detrimental for responses to chronic NIC exposure (Figure 1). To recapitulate this scenario, cultured renal proximal tubule cells (NRK52E) were transfected with either a p66shc plasmid ( $\uparrow$ p66shc), with a short-hairpin MnSOD plasmid ( $\downarrow$ MnSOD), or with an Nrf2 siRNA ( $\downarrow$ Nrf2) plasmid to overexpress p66shc or knockdown MnSOD or Nrf2 expression, respectively. Control cells were transfected with a control plasmid or with a scrambled siRNA. These cells were treated with either 2 or $200 \mu \mathrm{M} / 1 \mathrm{NIC}$. Extent of ROS production was determined and compared with untreated cells. Figure $\mathbf{4 a}$ demonstrates that although $2 \mu \mathrm{M} / \mathrm{l}$ NIC does not increase ROS production, overexpression of p66shc and knockdown of MnSOD (Figure 2a) or Nrf2 (Figure 2b) augmented it. Using $200 \mu \mathrm{M} / \mathrm{l}$ NIC significantly increased ROS production as reported previously (19), the extent of which was further increased by overexpressing

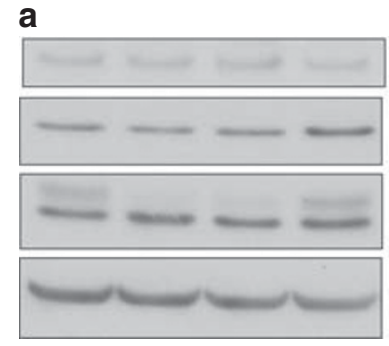

Adolescent

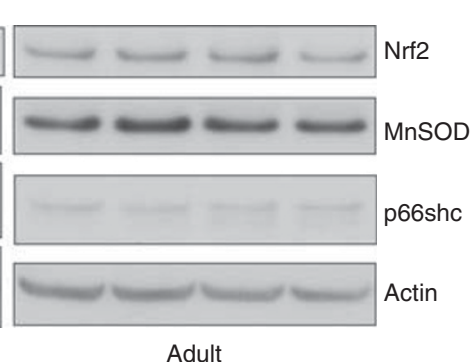

b

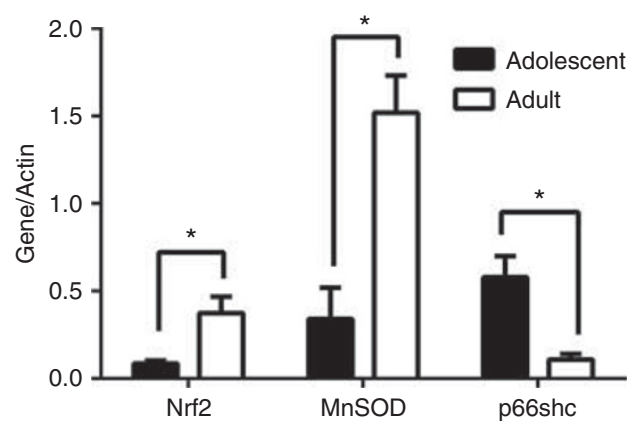

Figure 3. Pretreatment levels of select antioxidant (Nrf2, MnSOD) and pro (p66shc)-oxidant genes in the adolescent kidney differ from those in the adult kidney. (a) Expression of Nrf2, MnSOD, and p66shc was determined by Western blotting in the kidneys of untreated mice, as described in Figure 1 by Western blotting. Equal loading was assessed by reprobing the blots with an anti-actin antibody. Blots shown are representatives from 6 animals. (b) Densitometric analysis of the Western blots. $n=6 ;{ }^{*} P<0.05$ as indicated. 

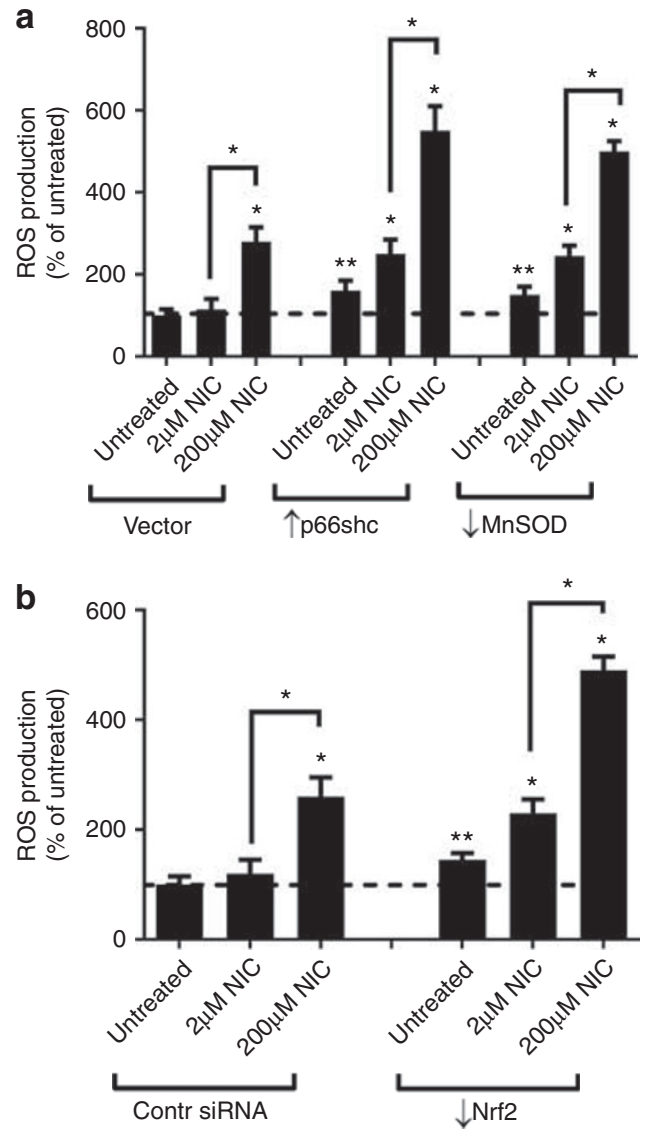

Figure 4. Overexpression of p66shc or knockdown of MnSOD or Nrf2 augments nicotine-induced ROS release in cultured renal proximal tubule cells. (a) NRK52E cells were transfected with a p66shc-expressing ( $\uparrow$ p66shc) plasmid or a short-hairpin MnSOD ( $\downarrow$ MnSOD) plasmid to overexpress or knockdown p66shc or MnSOD, respectively. A set of cells were transfected with an empty vector. Cells were left untreated or treated with $2 \mu \mathrm{m} / \mathrm{l}$ or $200 \mu \mathrm{m} / \mathrm{l}$ nicotine, and ROS release was determined. ROS production was expressed as $\%$ of the untreated value. $n=3 ;{ }^{*} P<0.05$ compared with untreated or as indicated; ${ }^{*} P<0.05$ compared with untreated vector control. (b) NRK52E cells were transfected with an Nrf2 siRNA ( $\downarrow N$ Nrf2) to knockdown Nrf2. A set of cells were transfected with a control (scrambled) siRNA. Cells were left untreated or treated with 2 or $200 \mu \mathrm{m} / \mathrm{I}$ nicotine, and ROS release was determined. ROS production was expressed as $\%$ of the untreated value. $n=3 ;{ }^{*} P<0.05$ compared with untreated or as indicated; $* * P<0.05$ compared with the untreated control siRNAtransfected group.

p66shc or knockdown of MnSOD or Nrf2 (Figure 2a,b). Importantly, p66shc overexpression or Nrf2/MnSOD knockdown itself increased endogenous production of ROS (Figure 4). These results suggest that high pretreatment levels of the pro-oxidant p66shc or low levels of the antioxidant $\mathrm{Nrf2/MnSOD}$ genes are maladaptive, which may explain the observed age-dependent responses to NIC exposure (Figure 1).

\section{DISCUSSION}

Smoking is a major risk factor in progression of chronic kidney diseases (20) in the pediatric population (21). A major component of cigarette smoke is NIC (2), which connects smoking to renal injury via inducing oxidative stress (3). Major sources of NIC exposure in the pediatric population are active smoking (22), e-cigarette use (5), and second-hand smoke exposure (9). Especially important is the increasing popularity of e-cigarettes, as it is considered a safe alternative to cigarettes (6). In reality, the peak NIC concentration achieved by e-cigarettes is similar to that in tobacco cigarettes (23) and hence, they represent similar renal risk as tobacco cigarettes.

Although the role of active smoking in renal risk is recognized, the role of second-hand smoke is overlooked (21). Experimental studies showed that rats exposed to passive smoking not only develop renal oxidative stress and fibrosis (24) but are also more sensitive to oxidative stress and related fibrosis caused by other environmental pollutants (24). Also, second-hand smoke exposure may be a critical factor in chronic kidney disease progression in children (25).

Our studies show that active smoking-equivalent NIC exposure significantly increases oxidative stress and tubular injury in both age groups, although significantly more in the adolescent kidney (Figure 1). In contrast, passive smokingequivalent NIC exposure incites oxidative stress and consequent tubular damage only in the adolescent but not in the adult kidney (Figure 1). This, however, does not rule out that longer exposure may actually lead to oxidative stress in the adult kidney, too. Further studies are needed to confirm this possibility. Regardless, it is clear that the adult kidney is less sensitive to both active and passive smoking-equivalent NIC exposure and consequent oxidative stress than the adolescent kidney. It has to be pointed out that regardless of the existence of tubular injury (Figure 1c,d, neither concentrations of NIC cause renal dysfunction (Figure 1e,f). This is in agreement with our previous studies which showed that NIC alone causes only sublethal injury in the kidney without affecting its histology and function $(4,26)$.

One possible scenario that explains this age-dependent differential response is that the adolescent and, hence, the adolescent kidney may accumulate higher amounts of NIC than the adult mice/kidney. Cotinine-a stable NIC metabolite (2) -is the most widely used biomarker of tobacco exposure $(9,27)$ and cotinine level in the blood correlates with the extent of renal injury (28). Our study shows that levels of cotinine in the plasma and in the kidney are, not surprisingly, significantly higher after active than after passive smokingequivalent NIC exposure both in adolescents and adults (Figure 2). However, there are no differences in cotinine levels between adolescents and adults; hence, a differential NIC/cotinine accumulation is probably not a factor in differential responses to NIC exposure (Figure 1). On the other hand, we cannot rule out the possibility that NIC content may actually differ between adolescents and adults. Dempsey et al. (29) suggested that the half-life of NIC is higher in neonates' blood than in adults' (the kidney was not studied) even though neonates have the same cotinine halflife as adults (29). Whether a tentatively higher NIC level in 


\section{Nicotine and the adolescent kidney $\mid$ Articles}

the adolescent plasma/kidney is responsible for the observed differences depicted in Figure 1 needs further studies.

Another possibility is that the adolescent kidney has an immature oxidant-handling system. Studies showed that the expression of select antioxidant genes is lower in the developing rat kidney (12), whereas the levels of $\mathrm{H}_{2} \mathrm{O}_{2}$ producing enzymes are higher in the chick kidney (13). Previously, we have shown that the pro-oxidant p66shc gene is pivotal in generating renal oxidative stress by regulating mitochondrial ROS production (17). The transcription factor Nrf2 is important in antioxidant responses in the kidney (30) via transcriptional activation of select antioxidant genes, such as MnSOD (31).

Our present study establishes that the expression of p66shc (Figure 3) is higher whereas the levels of Nrf2 and MnSOD are lower in the adolescent than in the adult kidney (Figure 3). In addition, oxidative stress (HNE content) of the untreated adolescent kidney is also higher than that in the adult kidney (Figure 1). Altogether, these results predict a (more pro-oxidant) milieu in the adolescent kidney that is unfavorable for chronic NIC exposure. We hypothesized that this (pro-oxidant) milieu sensitizes the adolescent kidney to (1) passive smoking-equivalent NIC exposure (Figure 1a) and (2) a more severe response to active smoking-equivalent NIC exposure (Figure $\mathbf{1 b}$ ). In contrast, the adult kidney is resistant to passive smoking-equivalent NIC exposure and also less sensitive to active smoking-equivalent NIC (Figure 1). In vitro, we could recapitulate this scenario by challenging renal proximal tubule cells with low $(2 \mu \mathrm{M} / \mathrm{l})$ or high $(200 \mu \mathrm{M} / \mathrm{l}) \mathrm{NIC}$ to simulate passive or active smoking. Figure 4 shows that low concentration of NIC does not incite ROS release in contrast to high NIC concentration in agreement with our in vivo results. However, overexpression of p66shc or knockdown of MnSOD or Nrf2 (condition that exists in the adolescent kidney) rendered the cells to produce a significant amount of ROS even at low NIC concentration and augmented high-dose NIC-mediated ROS release (Figure 4a). We also found that overexpression of p66shc or knockdown of MnSOD or Nrf2 increased endogenous (untreated) ROS release (Figure 4a,b), which is similar to the condition found in the adolescent kidneys (Figure 1).

Whether the unfavorable environment in the adolescent kidney to NIC exposure is due to the pre-existing oxidative stress or other impact of high p66shc but low Nrf2/MnSOD levels needs further investigation. It is noteworthy that low plasma antioxidant capacity predicts high incidence of oxidative stress-related diseases in infants (32). Nrf2 (33) or MnSOD (34) deficiency, or high level of p66shc (19) results in a pre-existing oxidative stress that amplifies oxidative stress due to a secondary stressor. Active smoking-equivalent chronic NIC exposure via increasing oxidative stress $(4,35)$ sensitizes the kidney to respond more severely to a secondary insult such as acute (4) or chronic (36) injury in various animal models and in the renal patients (20).

These results call attention to a potentially enhanced renal risk in response to active (tobacco or e-cigarette smoking) or second-hand smoke exposure in adolescents compared with that in adults.

\section{STATEMENT OF SUPPORT}

This work was supported by a grant from the Department of Pediatrics, University of Mississippi Medical Center, and the Bower Foundation

Disclosure: The authors declare no conflict of interest.

\section{REFERENCES}

1. Orth SR. Smoking-a renal risk factor. Nephron 2000;86:12-26.

2. Benowitz NL, Hukkanen J, Jacob P 3rd. Nicotine chemistry, metabolism, kinetics and biomarkers. Handb Exp Pharmacol 2009: 29-60.

3. Jaimes EA, Tian RX, Raij L. Nicotine: the link between cigarette smoking and the progression of renal injury? Am J Physiol Heart Circ Physiol 2007;292:H76-82.

4. Arany I, Grifoni S, Clark JS, Csongradi E, Maric C, Juncos LA. Chronic nicotine exposure exacerbates acute renal ischemic injury. Am J Physiol Renal Physiol 2011;301:F125-33.

5. Lauterstein D, Hoshino R, Gordon T, Watkins BX, Weitzman M, Zelikoff J. The changing face of tobacco use among United States youth. Curr Drug Abuse Rev 2014;7:29-43.

6. Baeza-Loya S, Viswanath H, Carter A, et al. Perceptions about e-cigarette safety may lead to e-smoking during pregnancy. Bull Menninger Clin 2014;78:243-52.

7. Preventing tobacco use among young people. A report of the Surgeon General. Executive summary. MMWR Recomm Rep 1994;43:1-10.

8. Collaco JM, Drummond MB, McGrath-Morrow SA. Electronic cigarette use and exposure in the pediatric population. JAMA Pediatr 2015;169: 177-82.

9. Omoloja A, Tyc VL. Tobacco and the pediatric chronic kidney disease population. Pediatr Nephrol 2015;30:235-43.

10. Puig C, Garcia-Algar O, Monleon T, et al. A longitudinal study of environmental tobacco smoke exposure in children: parental self reports versus age dependent biomarkers. BMC Public Health 2008;8:47.

11. Thaqi A, Franke K, Merkel G, Wichmann HE, Heinrich J. Biomarkers of exposure to passive smoking of school children: frequency and determinants. Indoor Air 2005;15:302-10.

12. Gupta A, Gupta A, Nigam D, Shukla GS, Agarwal AK. Profile of reactive oxygen species generation and antioxidative mechanisms in the maturing rat kidney. J Appl Toxicol 1999;19:55-9.

13. Zemanova Z, Gossrau R. Development of hydrogen peroxide (H2O2)generating oxidases in chick organs with special reference to kidneys. Acta Histochem 1997;99:37-45.

14. Wilking JA, Hesterberg KG, Nguyen VH, Cyboron AP, Hua AY, Stitzel JA. Comparison of nicotine oral consumption and baseline anxiety measures in adolescent and adult $\mathrm{C} 57 \mathrm{BL} / 6 \mathrm{~J}$ and $\mathrm{C} 3 \mathrm{H} / \mathrm{Ibg}$ mice. Behav Brain Res 2012;233:280-7.

15. Tang D, Warburton D, Tannenbaum SR, et al. Molecular and genetic damage from environmental tobacco smoke in young children. Cancer Epidemiol Biomarkers Prev 1999;8:427-31.

16. van Timmeren MM, van den Heuvel MC, Bailly V, Bakker SJ, van Goor H, Stegeman CA. Tubular kidney injury molecule-1 (KIM-1) in human renal disease. J Pathol 2007;212:209-17.

17. Arany I, Faisal A, Clark JS, Vera T, Baliga R, Nagamine Y. p66SHCmediated mitochondrial dysfunction in renal proximal tubule cells during oxidative injury. Am J Physiol Renal Physiol 2010;298:F1214-21.

18. Arany I, Hall S, Reed DK, Dixit M. The pro-oxidant gene p66shc increases nicotine exposure-induced lipotoxic oxidative stress in renal proximal tubule cells. Mol Med Rep 2016;14:2771-7.

19. Arany I, Clark J, Reed DK, Juncos LA. Chronic nicotine exposure augments renal oxidative stress and injury through transcriptional activation of p66shc. Nephrol Dial Transplant 2013;28:1417-25.

20. Orth SR, Hallan SI. Smoking: a risk factor for progression of chronic kidney disease and for cardiovascular morbidity and mortality in renal 


\section{Articles | Arany et al.}

patients-absence of evidence or evidence of absence? Clin J Am Soc Nephrol 2008;3:226-36.

21. Tasic V, Janchevska A, Emini N, Sahpazova E, Gucev Z, Polenakovic M. Chronic kidney disease - pediatric risk factors. Pril (Makedon Akad Nauk Umet Odd Med Nauki) 2016;37:9-13.

22. Garcia-Esquinas E, Loeffler LF, Weaver VM, Fadrowski JJ, Navas-Acien A. Kidney function and tobacco smoke exposure in US adolescents. Pediatrics 2013;131:e1415-23.

23. Marsot A, Simon N. Nicotine and cotinine levels with electronic cigarette: a review. Int J Toxicol 2016;35:179-85.

24. Boor P, Casper S, Celec P, et al. Renal, vascular and cardiac fibrosis in rats exposed to passive smoking and industrial dust fibre amosite. J Cell Mol Med 2009;13:4484-91.

25. Omoloja A, Jerry-Fluker J, Ng DK, et al. Secondhand smoke exposure is associated with proteinuria in children with chronic kidney disease. Pediatr Nephrol 2013;28:1243-51.

26. Arany I, Hall S, Reed DK, Reed CT, Dixit M. Nicotine enhances high-fat diet-induced oxidative stress in the kidney. Nicotine Tob Res 2016;18: 1628-34.

27. Hukkanen J, Jacob P III, Benowitz NL. Metabolism and disposition kinetics of nicotine. Pharmacol Rev 2005;57:79-115.

28. Kim KY, Lee YJ, Chung BC, Hong J, Jung BH. Relations between toxicity and altered tissue distribution and urinary excretion of nicotine, cotinine, and hydroxycotinine after chronic oral administration of nicotine in rats. Drug Chem Toxicol 2010;33:166-72.

29. Dempsey D, Jacob P 3rd, Benowitz NL. Nicotine metabolism and elimination kinetics in newborns. Clin Pharmacol Ther 2000;67:458-65.

30. Zoja C, Benigni A, Remuzzi G. The Nrf2 pathway in the progression of renal disease. Nephrol Dial Transplant 2013;29(Suppl 1):i19-24.

31. Macmillan-Crow LA, Cruthirds DL. Invited review: manganese superoxide dismutase in disease. Free Radic Res 2001;34:325-36.

32. Wijnberger LD, Krediet TG, Visser GH, van Bel F, Egberts J. Early neonatal antioxidant capacity after preexisting impaired placental function. Early Hum Dev 2003;71:111-6.

33. Kim HJ, Vaziri ND. Contribution of impaired Nrf2-Keap1 pathway to oxidative stress and inflammation in chronic renal failure. Am J Physiol Renal Physiol 2010;298:F662-71.

34. Thompson KH, Godin DV, Lee M. Tissue antioxidant status in streptozotocin-induced diabetes in rats. Effects of dietary manganese deficiency. Biol Trace Elem Res 1992;35:213-24.

35. Orth SR, Viedt C, Ritz E. Adverse effects of smoking in the renal patient. Tohoku J Exp Med 2001;194:1-15.

36. Rezonzew G, Chumley P, Feng W, Hua P, Siegal G, Jaimes EA. Nicotine exposure and the progression of chronic kidney disease: Role of the alpha7-nicotinic acetylcholine receptor. Am J Physiol Renal Physiol 2012;303:F304-12. 